\title{
Managing Crohn's Disease in The Older Adult: A Case Study Approach
}

\author{
Andrea Jennings Dr.PH., RN* \\ Senior Nurse Researcher, Geriatric Research Education and Clinical Center, Ohio, USA \\ *Corresponding author: Andrea Jennings, Senior Nurse Researcher, Geriatric Research Education and Clinical Center, VA Northeast \\ Ohio Healthcare System, 10701 East Boulevard, Cleveland, Ohio 44106, USA.
}

To Cite This Article: Andrea Jennings, Managing Crohn's Disease in The Older Adult: A Case Study Approach. 2020 - 9(4). AJBSR.MS.ID.001403. DOI: 10.34297/AJBSR.2020.09.001403.

Received: 眥 June 26, 2020; Published: 鰏 July 07, 2020

\begin{abstract}
Crohn's Disease is a chronic bowel disease that can affect any part of the gastrointestinal tract. Both genetics and environmental factors may contribute to a diagnosis of Crohn's Disease. This case study highlights how older adults with Crohn's Disease may face different challenges compared to younger populations. A multidisciplinary healthcare team is needed to initiate a plan of care for this vulnerable population. With ongoing evaluation of the plan of care, positive health outcomes may be likely to occur for older adults with Crohn's Disease.
\end{abstract}

\section{Introduction}

Crohn's Disease is defined as a bowel disease that is chronic and can affect any part of the gastrointestinal tract [1]. Crohn's Disease may manifest by inheriting genes that causes the immune system to malfunction as it reacts to specific environmental factors [1]. These environmental factors may include chemicals, stress, air pollutants, viruses, type of diet, and utilization of antibiotics, however additional studies are needed to unequivocally link these factors to Crohn's Disease [1-2]. Cases of Crohn's Disease for those individuals 60 and over has increased over the years [3]. A diagnosis of Crohn's Disease for older adults is often delayed significantly given symptoms from existing conditions that may be masking the symptoms of Crohn's Disease [3]. Studies have revealed that older adults diagnosed with Crohn's Disease may be more susceptible to infections, malnutrition, malignancy, increased rates of mortality due to surgeries, and polypharmacy [4,5]. Thus, treatment goals for older adults with Crohn's will vary in comparison to younger populations with this disease. The following case study highlights how the management of Crohn's Disease can present challenges to the older adult and to the health care team.

\section{Case Study}

Mr. Smith is a 64 year - old married man who was just diagnosed with Crohn's Disease in an outpatient clinic. A colonoscopy and upper endoscopy was performed to confirm the Crohn's Disease diagnosis. Mr. Smith was diagnosed with ileitis, which is a type of Crohn's Disease impacting the ileum. In the past five years, Mr. Smith has complained of bouts of diarrhea and abdominal pain and was misdiagnosed with infectious colitis. Mr. Smith has diabetes mellitus, arthritis, and a history of kidney cancer as he is taking medications for his diabetes and arthritis. His current symptoms include diarrhea, abdominal pain, and loss of appetite, fatigue, and vision changes. For years, he has had a steady weight loss and weighs 130 pounds. He has done some research on his own and was interested in having a fecal microbata transplantation for treatment. He lives in a suburban community and has access to health care. He is working full time and has health insurance from his company. Mr. Smith lives with his wife who also has multiple chronic conditions and they have a limited social support network. Mr. Smith is overwhelmed about this new diagnosis of Crohn's Disease as this is yet another disease that he has to manage. Mr. Smith has an upcoming appointment to see the nurse case manager to discuss his plan of care.

\section{Discussion}

The nurse case manager in this situation will need to use a multidisciplinary approach to coordinate the plan of care for $\mathrm{Mr}$. Smith. The fact that Mr. Smith has lost weight over the years and weighs 130 pounds is cause for concern. Exploring if there are vitamin deficiencies such as Vitamin B-12, Folic Acid, Vitamin D, 
Iron, Calcium, Zinc, and Magnesium will need to be investigated by the physician. The nutritionist will need to counsel Mr. Smith about modifying his diet and should inform him that diet plans are very individualized for Crohn's patients. A discussion regarding ways in which Mr. Smith can supplement his current diet with high caloric nutritional drinks is a crucial action that needs to be initiated by the nutritionist.

It is highly recommended that Mr. Smith's physician engage in shared decision making with regards to the different medication treatment regimens that are used for Crohn's Disease. Shared decision making occurs when health care team members along with the patient are actively involved in making treatment decisions based on best clinical practices [6]. Mr. Smith is 65 years old, has preexisting chronic conditions, is taking medications for these conditions, has a history of kidney cancer, and has some mobility problems. These are all factors that can impact what type of medication regimen is prescribed and selected. Since Mr. Smith has inquired about fecal microbata transplantation for treatment, he should be informed by his physician that this procedure is not FDA (The US Food and Drug Administration) approved as of yet [7]

The nurse case manager should arrange for an ophthalmology consult given Mr. Smith's change in vision. A comprehensive examination of Mr. Smith's informal and formal support systems is essential as this is an integral component of managing this disease. Encouraging Mr. Smith and his wife to join a support group would be beneficial for them both. The Crohn's and Colitis Foundation is a valuable resource and will allow Mr. Smith and his wife to access educational materials that is based on best clinical practices. Mr. Smith should be monitored for signs of depression and anxiety disorders over time and referred appropriately to the appropriate mental health professional. A social work consult may be necessary in this case because Mr. Smith will need to know about financial assistance programs that are available for the costly medication treatments that he may have to select from. Due to the anxiety that Mr. Smith is experiencing, the nurse case manager should discuss a variety of coping strategies with him and direct him to resources that are available regarding these strategies.

\section{Conclusion}

Recognizing the various challenges that may exist for older patients diagnosed with Crohn's Disease is a step in the right direction for health care providers. Health care providers need to be aware of the medication treatment regimens and cognizant of the resources that may need to be activated by older adults with Crohn's Disease. A multidisciplinary approach is essential when developing, implementing, and evaluating the plan of care for older adults with Crohn's Disease. The ultimate goal is to control the symptoms that older adults are presenting with to assure a productive life.

\section{Reference}

1. Crohn's and Colitis Foundation Living with Crohn's Disease. 2018.

2. Shuk-Mei Ho, Lewis JD, Mayer, EA, Bernstein CN, Emil Chuang, et al. (2019) Challenges in IBD research: Environmental triggers. Inflammatory Bowel Disease 25(Suppl 2): S13-S23.

3. Le Blanc J-F, Wiseman, D, Lakatos PL, Bessissow T (2019) Elderly patients with inflammatory bowel disease. Updated review of therapeutic landscape. World Journal of Gastroenterology 25(30): 4158-4171.

4. Ananthakrishnan AN, Binion DG (2009) Treatment of ulcerative colitis in the elderly. Dig Dis 27(3): 327-334.

5. Bollegala N, Jackson TD, Nguyen GC (2016) Increased postoperative mortality and complications among elderly patients with inflammatory bowel diseases: An analysis of the National Surgical Quality Improvement Program Cohort. Clin Gastroenterol Hepatol 14(9): 1274-1281.

6. Elwyn G, Frosch D, Thomson R, Natalie Joseph-Williams, Amy Lloyd, et al. (2012) Shared decision making: A model for clinical practice. J Gen Intern Med 27: 1361-1367.

7. Nilesh C, Costello SP, Limketkai BN, Claire E Parker, Tran M Nguyen, et al. (2020) Alternative and complementary approaches for the treatment of inflammatory bowel disease: Evidence from Cochrane Reviews. Inflamm Bowel Dis 26(6): 843-851. 